\title{
Sampling Error: Impact on the Quantitative Analysis of Nanoparticle-based Surface-enhanced Raman Scattering Immunoassays
}

Alexis C. Crawford, ${ }^{\mathrm{a}, \mathrm{b}}$ Aleksander Skuratovsky, ${ }^{\mathrm{b}, \mathrm{c}}$ and Marc D. Porter ${ }^{\mathrm{a}, \mathrm{b}, \mathrm{c} *}$

${ }^{a}$ Department of Chemistry, University of Utah, Salt Lake City, UT 84112

${ }^{\mathrm{b}}$ The Nano Institute of Utah

${ }^{c}$ Department of Chemical Engineering, University of Utah, Salt Lake City, UT 84112

* Corresponding author (marc.porter@utah.edu) 


\section{Table of Contents}

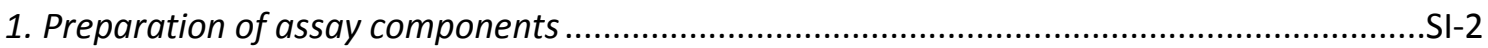

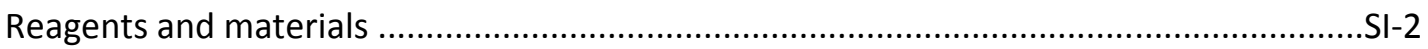

Preparation of extrinsic Raman labels (ERLs) …........................................................

Preparation of capture substrate and SERS immunoassay procedure ................................SI-3

2. SEM image of completed SERS immunoassay substrate .......................................................

3. Determination of the sampling constant $\left(K_{S}\right)$ for the simulated assay .................................SI-4

4. Figures

Figure S1

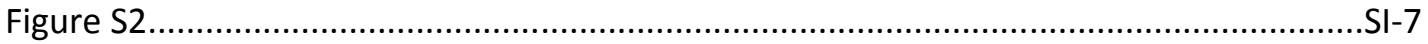

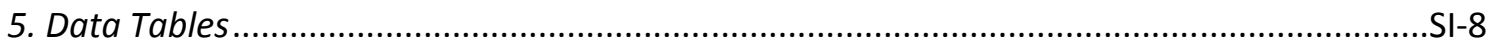

Table S1. AAR and corresponding spot sizes and areas for 3 and $2 \mathrm{~mm}$ substrates ...........SI-8

Table S2. Listing of data presented in Figure 3 and Figure 4 ...........................................

Table S3. Listing of data from calculations using Equation 3............................................

Table S4. Listing of data presented in Figure 5 with absolute and relative error given

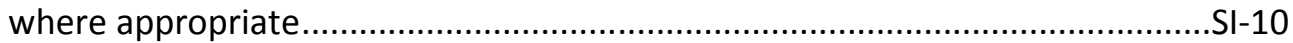

Table S5. Listing of data presented in Figure 7a and 7b ....................................................

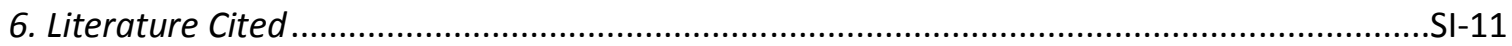




\section{Preparation of assay components}

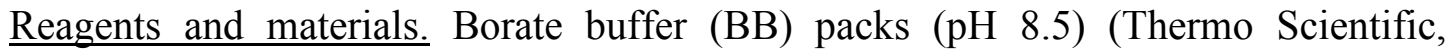
Wilmington, DE), modified Dulbecco's phosphate-buffed saline packs (pH 7.4) (Thermo Scientific, Wilmington, DE), dithiobis(succinimidyl propionate) (DSP) (Thermo Scientific, Wilmington, DE), StartingBlock ${ }^{\circledR}$, goat anti-human IgG (anti-human IgG) (Pierce, Wilmington, DE), and H-IgG (Pierce, Wilmington, DE) were used as received. Acetonitrile (ACN) (Sigma Aldrich, St. Louis, MO), Tween 20 (Sigma Aldrich, St. Louis, MO), sodium chloride (Sigma Aldrich, St. Louis, MO), octadecanethiol (ODT) (Sigma Aldrich, St. Louis, MO), and bovine serum albumin (BSA) (Sigma Aldrich, St. Louis, MO) were used as received. Epoxy 377 (EPO-TEK, Billerica, MA) poly(dimethyl siloxane) (PDMS) (SlyGard, Midland, MI); 200-proof ethanol (Pharmco-AAPER, Shelbyville, KY); and gold nanoparticles (AuNPs) (Ted Pella via BBI Solutions, Cardiff, UK) were also used without further purification. The synthesis of the Raman reporter molecule 5-5'-dithiobis(succinimidyl-2-nitrobenzoate) (DSNB) has been described previously. ${ }^{1}$

Preparation of extrinsic Raman labels (ERLs). A detailed description of the SERS assay procedure has been appeared previously. ${ }^{2}$ The ERLs were prepared in batches using a $0.96-\mathrm{mL}$ suspension of $60-\mathrm{nm}$ AuNPs at $2.6 \times 10^{10} \mathrm{AuNPs} / \mathrm{mL}$, and adjusting the buffer strength by the addition of $40 \mu \mathrm{L}$ of $50 \mathrm{mM} \mathrm{BB}$ (pH 8.5). The buffered AuNPs (2.0 $\mathrm{mM} \mathrm{BB}$ ) were then modified by the addition of $10 \mu \mathrm{L}$ of $1.0 \mathrm{mM}$ DSNB, followed by $10.0 \mu \mathrm{g}$ of anti-human $\operatorname{IgG}(13.3 \mu \mathrm{L}$ of $1.5 \mathrm{mg} / \mathrm{mL}$ stock solution), and $100 \mu \mathrm{L}$ of $10 \%$ BSA (20 mM BB). After letting the resulting suspension stand for $7 \mathrm{~h}$, excess reactants were removed by centrifugation at 2,000g for $10 \mathrm{~min}$ to pellet the ERLs and the careful 
withdraw of the supernatant. The ERLs were resuspended with $1.0 \mathrm{~mL} \mathrm{1 \%} \mathrm{BSA} \mathrm{(2.0} \mathrm{mM}$ BB). This cleanup process was repeated two more times. As a result of these steps, the ERLs were concentrated to $4.0 \times 10^{10}$, as determined using the spectrometric method of Haiss, et al. ${ }^{3}$

Preparation of the capture substrate and SERS immunoassay procedure. The capture substrate was prepared on template-stripped gold (TSG) by first creating a hydrophobic boundary, which defined an address diameter $(2.0$ or $3.0 \mathrm{~mm})$ by using microcontact printing with ODT. The address was then reacted with DSP (14-16 h), followed by 2.0 $\mu \mathrm{g} / \mathrm{mL}$ of the capture antibody, anti-human $\mathrm{IgG}$, in $10 \mathrm{mM}$ phosphate-buffered saline with $1 \%$ Tween 20 (PBST, $\mathrm{pH}$ 7.4) for $7 \mathrm{~h}$. These capture substrates were rinsed three times with PBST and treated with StartingBlock ${ }^{\circledR}$. Finally, the substrates were incubated with antigen solution $(20 \mu \mathrm{L})$, rinsed three times with PBST, inverted, exposed to the ERL suspension for $16 \mathrm{~h}$, rinsed with $\mathrm{BB}$ [0.1\% Tween 20, $10 \mathrm{mM} \mathrm{NaCl}$ (BBT, $\mathrm{pH}$ 8.5)], and allowed to dry under ambient conditions for $1-2 \mathrm{~h}$.

\section{SEM image of completed SERS immunoassay substrate}

This section describes the data, obtained from scanning electron microscopy (SEM) imaging, used to establish the true value applied in the random accumulation of antigen simulations The samples were imaged using a field emission scanning electron microscopy (NanoNova SEM), equipped with a through-the-lens detector. The images were analyzed with imaging ImageJ software (National Institutes of Health, Bethesda, MD). 
A representative image of the SERS immunoassay substrate is shown in Figure S1. This image is for a capture substrate that was first exposed to H-IgG antigen at a concentration of $6.67 \times 10^{-11} \mathrm{M}\left(10.0 \mathrm{ng} \mathrm{mL}^{-1}\right)$ and subsequently to a suspension of ERLs.

The image consists largely of isolated ERLs, a small number of cluster-like (e.g., dimers, trimers, and short filaments) arrangements, and clearly visible voids. A few nonspherically shaped ERLs are also evident. This distribution is characteristic of randomly accumulated particles on a surface, and is representative of 5 images obtained from different locations across the sample surface. Determination of the number of ERLs in the 5 images yielded an average density of $13.5 \pm 1.5$ ERLs $\mu \mathrm{m}^{-2}$. For the Monte Carlo simulations, we used a lower PSA density (1.415 PSAs $\mu \mathrm{m}^{-2}$ or $1.000 \times 10^{7}$ PSAs for a 3$\mathrm{mm}$ diameter address) in order to manage computational time. Using a 1:1 proportionality, this corresponds to an $\mathrm{H}-\mathrm{IgG}$ concentration of $4.35 \times 10^{-11} \mathrm{M}(\sim 0.74$ $\mathrm{ng} / \mathrm{mL} \mathrm{H}-\mathrm{IgG})$. The true value for the computational simulation was defined to have 4 significant figures in order to more fully assess the impact of sampling on the results. We have assumed that each captured antigen is tagged by only one ERL.

\section{Determination of the sampling constant $\left(K_{S}\right)$ for the simulated assay}

The results of the model simulations (Figures 4 and 5) show that the accuracy of the results converge more rapidly towards the true value with increases in AAR, which is in accord with the expectations of the sampling problem often found when determining trace constituents in geological samples. ${ }^{4}$ Along these lines, Equation 2, which is often used to establish the mass of a sample required in geological analysis to reach a given accuracy and precision, was adapted to determine $K_{S}$ for the simulated SERS substrate by 
setting $m$ equal to AAR. This sampling problem comes about due to the highly sensitive nature of the signal distribution at these relatively small sample sizes for trace geological and SERS analysis methods.

To predict the $K_{S}$ value, 10 separate simulations were carried out and analyzed to determine the spread of the results for evenly spaced increments of AARs between $1.0 \times 10^{-3}$ to 0.5 . The resulting PSA densities versus AAR are presented in Figure S2a. The density distributions again exhibit an increase in the accuracy and precision with larger values of AAR. This plot was used to calculate values for the \%RSD of the measurement at each AAR, which was then applied to construct the graph in Figure $6 \mathrm{~b}$ of $\%$ RSD versus the diameter of the sampling area. For a $1 \%$ RSD, the laser spot size has a diameter of $550 \mu \mathrm{m}$, which corresponds to a $K_{S}$ of $3.4 \times 10^{-2}$ AAR. After finding $K_{S}$, Equation 2 can be rearranged to calculate the AAR required for a given RSD. By loosening the tolerance in the precision from $1 \%$ to $5 \% \mathrm{RSD}$, the required value of $\mathrm{AAR}$ decreases from $3.4 \times 10^{-2}$ to $1.4 \times 10^{-3}$ or from a sampling diameter of 560 to $110 \mu \mathrm{m}$, respectively. The results for the $5 \%$ RSD are of particular interest, being a more readily achievable laser spot diameter. A spot size of $100 \mu \mathrm{m}$, which is achievable in commercially available instrumentation, would require a laser power of $2 \mathrm{~W}$ to achieve an equivalent power density as a laser spot diameter of $5.0 \mu \mathrm{m}$ at $5 \mathrm{~mW}$. 


\section{Figures}

(a)

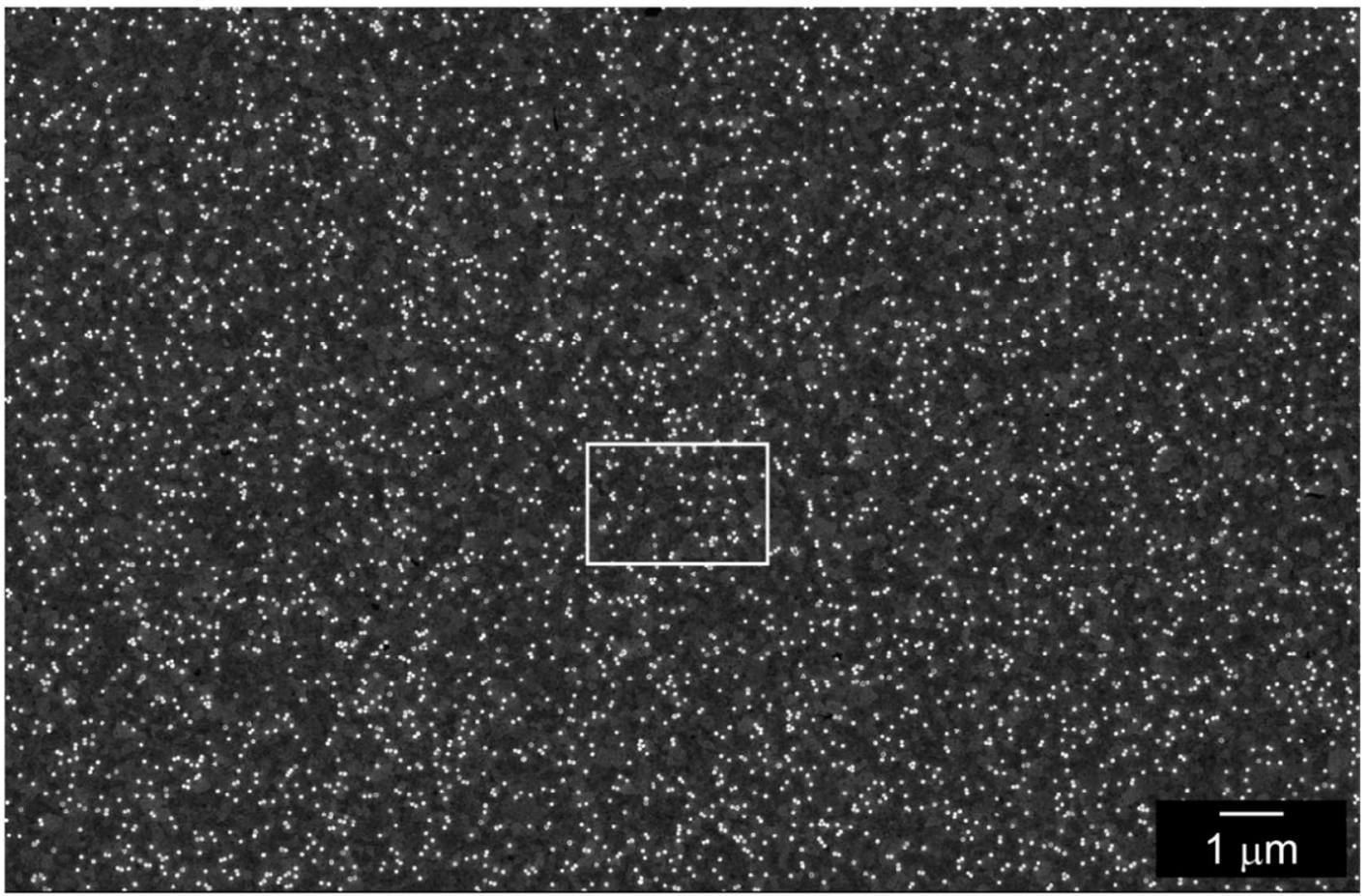

(b)

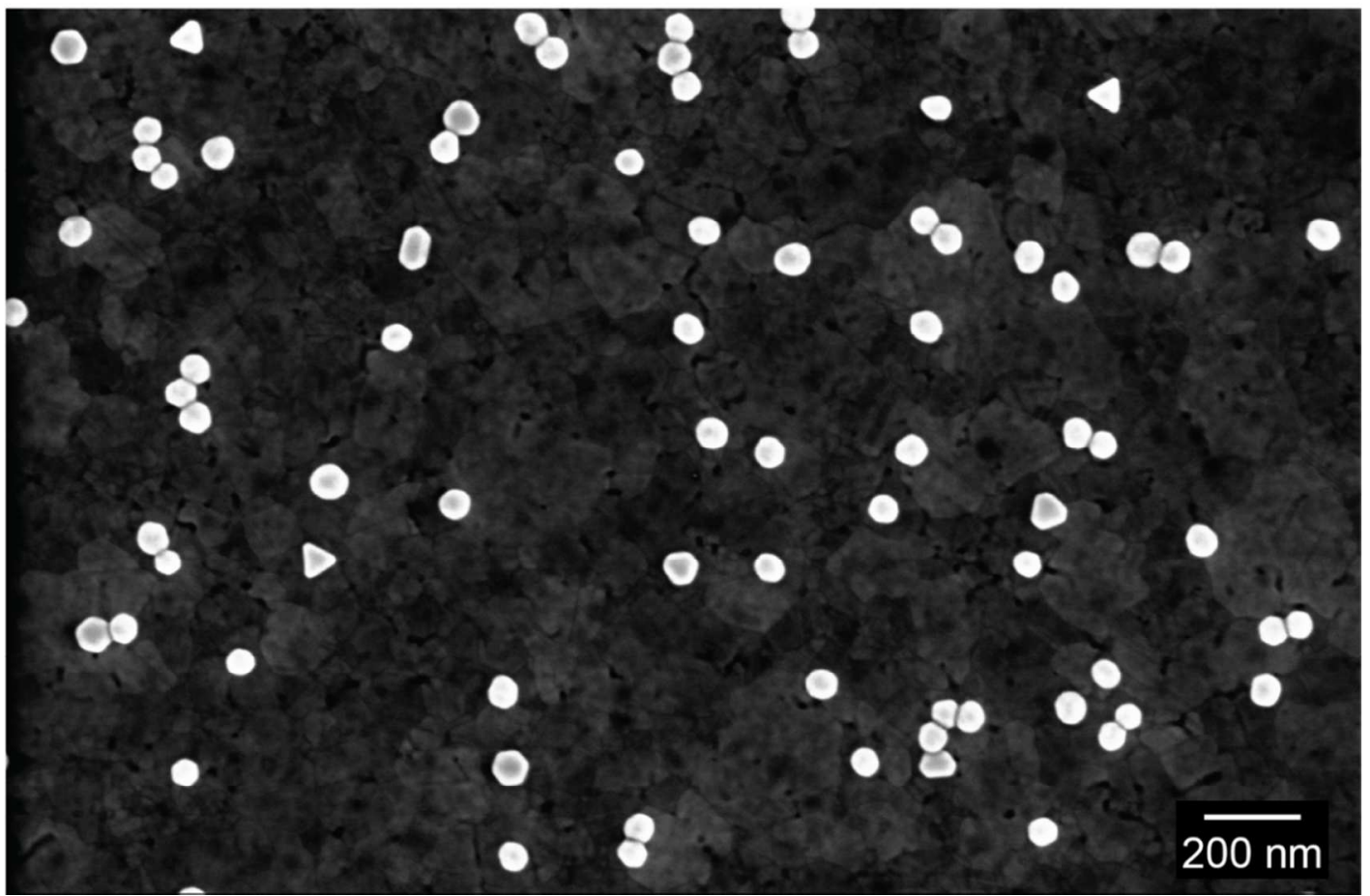

Figure S1. SEM image of a SERS immunoassay substrate for H-IgG at a concentration of $6.67 \times 10^{-11} \mathrm{M}\left(10.0 \mathrm{ng} \mathrm{mL}^{-1}\right)$, which has an ERL density of $\sim 13.5 \pm 1.5 \mathrm{ERLs} \mu \mathrm{m}^{-2}$. The brighter circular features in the SEM image are consistent with a $60-\mathrm{nm}$ AuNP core used to produce ERLs. (a) Image area of $\sim 290 \mu \mathrm{m}^{2}$; (b) enlargement of the highlighted area in the center of (a). 

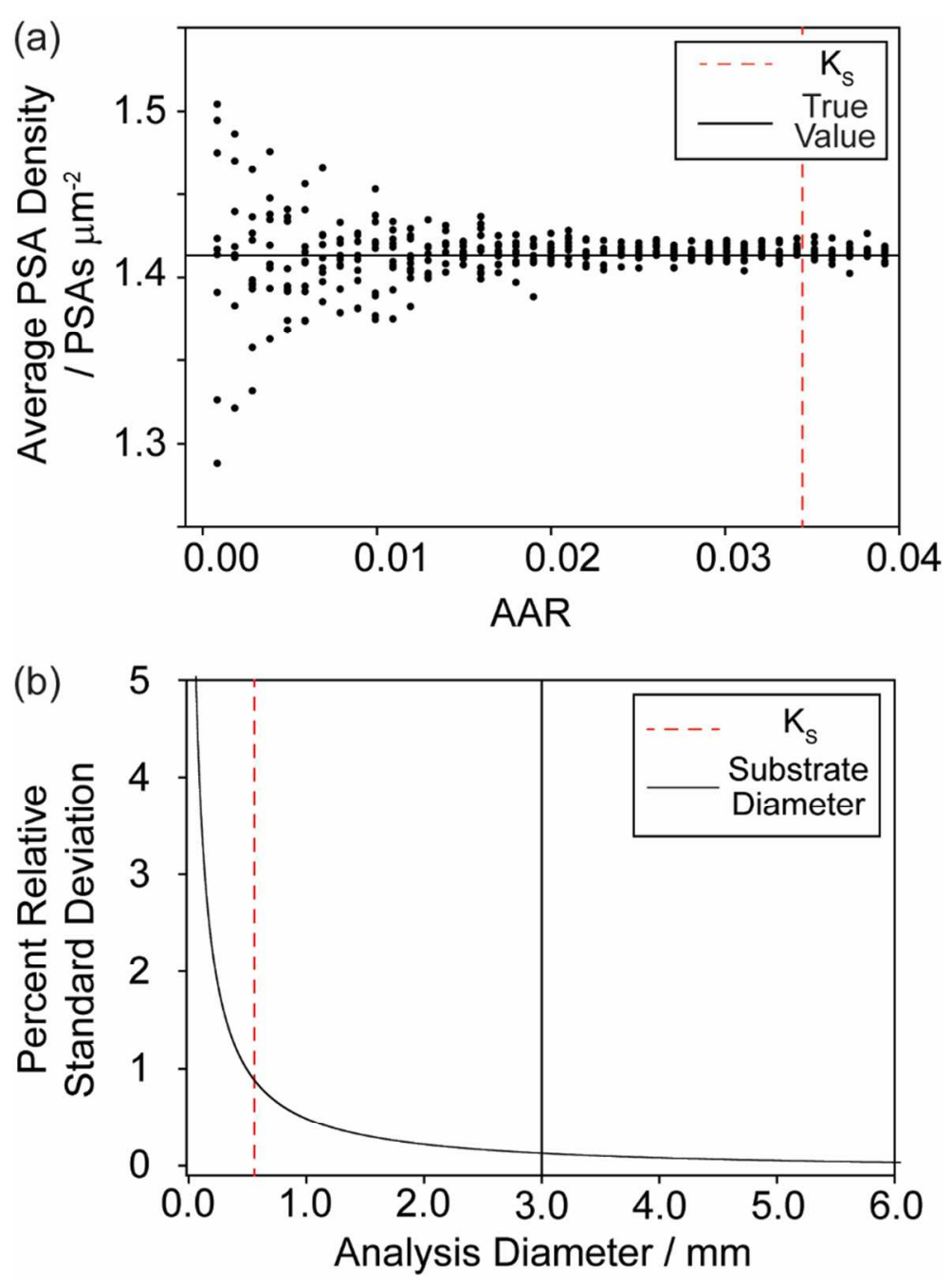

Figure S2. Determination of the sampling constant $\left(K_{S}\right)$ based on simulation results. (a) Sampling diagram of the results of the simulation for PSA enumeration of a pseudorandom distribution of PSAs on a $3.0-\mathrm{mm}$ substrate at 1.415 PSAs $\mu \mathrm{m}^{-2}$ and 10 simulation runs for an $n_{\text {replicate }}$ of one. (b) Plot of analysis diameter (mm) versus percent relative standard deviation equal to $\sqrt{K_{s} / m}$, based on Equation 2. The $y$-axis extends beyond the boundary of the $3.0-\mathrm{mm}$ address because the calculation assumes a substrate of infinite size. 


\section{Data Tables}

Table S1. AAR and corresponding spot size diameter and areas for 3 and $2 \mathrm{~mm}$ substrates.

\begin{tabular}{c|cc|cc}
\hline AAR & $\begin{array}{c}\text { Spot Size Diameter } \\
\text { for } 3 \mathrm{~mm} \text { substrate } \\
(\mu \mathrm{m})\end{array}$ & $\begin{array}{c}\text { Spot Size Area } \\
\text { for } 3 \mathrm{~mm} \\
\text { substrate }\left(\mathrm{mm}^{2}\right)\end{array}$ & $\begin{array}{c}\text { Spot Size Diameter } \\
\text { for } 2 \mathrm{~mm} \text { substrate } \\
(\mu \mathrm{m})\end{array}$ & $\begin{array}{c}\text { Spot Size Area } \\
\text { for } 2 \mathrm{~mm} \\
\text { substrate }\left(\mathrm{mm}^{2}\right)\end{array}$ \\
\hline 1.0 & $3.000 \times 10^{3}$ & 7.069 & $2.000 \times 10^{3}$ & 3.142 \\
0.99 & $2.985 \times 10^{3}$ & 6.998 & $1.990 \times 10^{3}$ & 3.110 \\
0.75 & $2.598 \times 10^{3}$ & 5.301 & $1.732 \times 10^{3}$ & 2.356 \\
0.50 & $2.121 \times 10^{3}$ & 3.534 & $1.414 \times 10^{3}$ & 1.571 \\
0.25 & $1.500 \times 10^{3}$ & 1.767 & $1.000 \times 10^{3}$ & 0.7854 \\
0.10 & $9.490 \times 10^{2}$ & 0.7069 & $6.325 \times 10^{2}$ & 0.3142 \\
$1.0 \times 10^{-2}$ & $3.000 \times 10^{2}$ & $7.069 \times 10^{-2}$ & $2.000 \times 10^{2}$ & $3.142 \times 10^{-2}$ \\
$1.0 \times 10^{-3}$ & 94.90 & $7.069 \times 10^{-3}$ & 63.25 & $3.142 \times 10^{-3}$ \\
$1.0 \times 10^{-4}$ & 30.00 & $7.069 \times 10^{-4}$ & 20.00 & $3.142 \times 10^{-4}$ \\
$1.0 \times 10^{-5 *}$ & 9.490 & $7.069 \times 10^{-5}$ & 6.325 & $3.142 \times 10^{-5}$ \\
$1.0 \times 10^{-6 *}$ & 3.000 & $7.069 \times 10^{-6}$ & 2.000 & $3.142 \times 10^{-6}$ \\
$1.0 \times 10^{-7 * *}$ & 0.9490 & $7.069 \times 10^{-7}$ & 0.6325 & $3.142 \times 10^{-7}$ \\
\hline
\end{tabular}

* Close to the laser spot size produced by the 10x objective.

** Close to the laser spot size produced by the 50x objective. 
Table S2. Listing of data presented in Figure 3 and Figure 4.

\begin{tabular}{|c|c|c|c|c|}
\hline AAR & $n_{\text {replicate }}$ & PSA density $\left(\# / \mu \mathrm{m}^{2}\right)$ & $\mathcal{D}$ avg $\left(\# / \mu m^{2}\right)$ & $s\left(\# / \mu \mathrm{m}^{2}\right)$ \\
\hline \multirow{10}{*}{$1.0 \times 10^{-5}$} & 1 & 1.4199 & 0.1528 & - \\
\hline & 2 & 1.3371 & 0.1293 & 0.1553 \\
\hline & 3 & 1.4231 & 0.1199 & 0.2002 \\
\hline & 4 & 1.4263 & 0.0545 & 0.2607 \\
\hline & 5 & 1.4397 & 0.0819 & 0.2189 \\
\hline & 10 & 1.4059 & 0.0257 & 0.2012 \\
\hline & 25 & 1.4256 & 0.0363 & 0.2114 \\
\hline & 50 & 1.4002 & 0.0342 & 0.2058 \\
\hline & 75 & 1.4094 & 0.0120 & 0.2158 \\
\hline & 100 & 1.4141 & 0.0157 & 0.2048 \\
\hline \multirow{10}{*}{$1.0 \times 10^{-6}$} & 1 & 1.2096 & 0.4386 & - \\
\hline & 2 & 1.2096 & 0.3749 & 0.5694 \\
\hline & 3 & 1.2945 & 0.4103 & 0.4464 \\
\hline & 4 & 1.3210 & 0.3148 & 0.6601 \\
\hline & 5 & 1.4006 & 0.2858 & 0.6302 \\
\hline & 10 & 1.4165 & 0.1659 & 0.6569 \\
\hline & 25 & 1.4120 & 0.1082 & 0.6358 \\
\hline & 50 & 1.3694 & 0.0476 & 0.6668 \\
\hline & 75 & 1.3666 & 0.0840 & 0.6704 \\
\hline & 100 & 1.4111 & 0.0322 & 0.6776 \\
\hline \multirow{10}{*}{$1.0 \times 10^{-7}$} & 1 & 2.3686 & 1.806 & - \\
\hline & 2 & 2.2286 & 0.8139 & 2.6636 \\
\hline & 3 & 1.4857 & 0.4952 & 1.8381 \\
\hline & 4 & 1.7510 & 0.7076 & 2.1357 \\
\hline & 5 & 1.5282 & 0.7923 & 1.9882 \\
\hline & 10 & 1.7192 & 0.6722 & 2.3966 \\
\hline & 25 & 1.5536 & 0.3820 & 2.3060 \\
\hline & 50 & 1.4263 & 0.2010 & 2.0160 \\
\hline & 75 & 1.2862 & 0.2702 & 2.0045 \\
\hline & 100 & 1.3690 & 0.1039 & 2.0824 \\
\hline
\end{tabular}


Table S3. Listing of data from calculations using Equation 3.

\begin{tabular}{ccccc}
\hline AAR & $S$ & $\% R S D$ & $n_{\text {replicate }}$ & $\%$ total area \\
\hline \multirow{2}{*}{0.99} & \multirow{2}{*}{$4.70 \times 10^{-5}$} & $1 \%$ & 1 & 0.99 \\
& & $5 \%$ & 1 & 0.99 \\
\hline \multirow{2}{*}{0.75} & \multirow{2}{*}{$2.94 \times 10^{-4}$} & $1 \%$ & 1 & 0.75 \\
& & $5 \%$ & 1 & 0.75 \\
\hline \multirow{2}{*}{0.50} & \multirow{2}{*}{$4.26 \times 10^{-4}$} & $1 \%$ & 1 & 0.50 \\
& & $5 \%$ & 1 & 0.50 \\
\hline \multirow{2}{*}{0.25} & \multirow{2}{*}{$8.62 \times 10^{-4}$} & $1 \%$ & 1 & 0.25 \\
& & $5 \%$ & 1 & 0.25 \\
\hline \multirow{2}{*}{0.10} & \multirow{2}{*}{$1.92 \times 10^{-3}$} & $1 \%$ & 1 & 0.10 \\
& & $5 \%$ & 1 & 0.10 \\
\hline \multirow{2}{*}{$1.0 \times 10^{-2}$} & \multirow{2}{*}{$6.63 \times 10^{-3}$} & $1 \%$ & 1 & $1.0 \times 10^{-2}$ \\
& & $5 \%$ & 1 & $1.0 \times 10^{-2}$ \\
\hline \multirow{2}{*}{$1.0 \times 10^{-3}$} & \multirow{2}{*}{$2.03 \times 10^{-2}$} & $1 \%$ & 1 & $1.0 \times 10^{-3}$ \\
& & $5 \%$ & 1 & $1.0 \times 10^{-3}$ \\
\hline \multirow{2}{*}{$1.0 \times 10^{-4}$} & \multirow{2}{*}{$6.51 \times 10^{-2}$} & $1 \%$ & 8 & $8.0 \times 10^{-4}$ \\
& & $5 \%$ & 3 & $3.0 \times 10^{-4}$ \\
\hline \multirow{2}{*}{$1.0 \times 10^{-5}$} & \multirow{2}{*}{0.209} & $1 \%$ & 857 & $8.6 \times 10^{-3}$ \\
& & $5 \%$ & 34 & $3.4 \times 10^{-4}$ \\
\hline \multirow{2}{*}{2.03} & $1 \%$ & 81,358 & $8.1 \times 10^{-3}$ \\
& \multirow{2}{*}{0.672} & $1 \%$ & 8768 & $8.8 \times 10^{-3}$ \\
& & $5 \%$ & 3,254 & $3.3 \times 10^{-4}$ \\
\hline
\end{tabular}

Table S4. Listing of data presented in Figure 5 with absolute and relative error given where appropriate.

\begin{tabular}{|c|c|c|c|c|c|c|c|c|}
\hline \multirow{2}{*}{ AAR } & \multirow{2}{*}{$\mu\left(\mathrm{PSAs} / \mu \mathrm{m}^{2}\right)$} & \multirow{2}{*}{$s\left(\mathrm{PSAs} / \mu \mathrm{m}^{2}\right)$} & \multicolumn{6}{|c|}{ error } \\
\hline & & & \multicolumn{3}{|c|}{ absolute* } & \multicolumn{3}{|c|}{ relative** } \\
\hline 0.75 & 1.415 & $4.43 \mathrm{E}-04$ & & & & & & \\
\hline 0.5 & 1.415 & 7.47E-04 & & & & & & \\
\hline 0.25 & 1.415 & $9.09 \mathrm{E}-04$ & & & & & & \\
\hline 0.10 & 1.415 & $2.22 \mathrm{E}-03$ & & & & & & \\
\hline $1.0 \times 10^{-2}$ & 1.414 & $6.01 \times 10^{-3}$ & $1.00 \times 10^{-3}$ & \pm & $6.00 \times 10^{-3}$ & $7.10 \times 10^{-2}$ & \pm & 0.424 \\
\hline $1.0 \times 10^{-3}$ & 1.419 & $2.63 \times 10^{-2}$ & $4.00 \times 10^{-3}$ & \pm & $2.60 \times 10^{-2}$ & 0.283 & \pm & 1.85 \\
\hline $1.0 \times 10^{-4}$ & 1.417 & $6.31 \times 10^{-2}$ & $2.00 \times 10^{-3}$ & \pm & $6.31 \times 10^{-2}$ & 0.141 & \pm & 4.56 \\
\hline $1.0 \times 10^{-5}$ & 1.370 & 0.220 & $4.50 \times 10^{-2}$ & \pm & 0.220 & 33.2 & \pm & 15.5 \\
\hline $1.0 \times 10^{-6}$ & 1.309 & 0.550 & 0.106 & \pm & 0.550 & 7.50 & \pm & 39.0 \\
\hline $1.0 \times 10^{-7}$ & ND & ND & & & & & & \\
\hline
\end{tabular}

* Given as the deviation and standard deviation of the results.

** Given as the absolute relative deviation and relative standard deviation of the results. 
Table S5. Listing of data presented in Figure 7a and 7b.

\begin{tabular}{ccccc}
\hline \multirow{2}{*}{$n_{\text {replicate }}$} & \multicolumn{2}{c}{$\mathcal{D}_{\text {Avg }}$} & \multicolumn{2}{c}{$S$} \\
\cline { 2 - 5 } & $0.5 \mu \mathrm{m}$ & $5.0 \mu \mathrm{m}$ & $0.5 \mu \mathrm{m}$ & $5.0 \mu \mathrm{m}$ \\
\hline 1 & 0.130 & $7.85 \times 10^{-2}$ & - & - \\
2 & 0.116 & $5.83 \times 10^{-2}$ & 0.160 & $6.38 \times 10^{-2}$ \\
3 & $9.00 \times 10^{-2}$ & $5.32 \times 10^{-2}$ & 0.228 & $4.52 \times 10^{-2}$ \\
4 & $7.97 \times 10^{-2}$ & $4.74 \times 10^{-2}$ & 0.214 & $3.13 \times 10^{-2}$ \\
5 & $7.89 \times 10^{-2}$ & $1.49 \times 10^{-2}$ & 0.158 & $6.26 \times 10^{-2}$ \\
10 & $4.45 \times 10^{-2}$ & $1.37 \times 10^{-2}$ & 0.178 & $5.31 \times 10^{-2}$ \\
25 & $4.75 \times 10^{-2}$ & $1.91 \times 10^{-2}$ & 0.174 & $4.98 \times 10^{-2}$ \\
50 & $2.11 \times 10^{-2}$ & $1.46 \times 10^{-2}$ & 0.178 & $4.26 \times 10^{-2}$ \\
75 & $1.52 \times 10^{-2}$ & $1.13 \times 10^{-2}$ & 0.177 & $4.73 \times 10^{-2}$ \\
100 & $8.25 \times 10^{-3}$ & $1.78 \times 10^{-3}$ & 0.177 & $4.80 \times 10^{-2}$ \\
\hline
\end{tabular}

\section{Literature Cited}

(1) Grubisha, D. S.; Lipert, R. J.; Park, H.-Y.; Driskell, J.; Porter, M. D., Femtomolar Detection of Prostate-Specific Antigen: An Immunoassay Based on SurfaceEnhanced Raman Scattering and Immunogold Labels. Anal Chem 2003, 75, 59365943.

(2) Driskell, J. D.; Kwarta, K. M.; Lipert, R. J.; Porter, M. D.; Neill, J. D.; Ridpath, J. F., Low-Level Detection of Viral Pathogens by a Surface-Enhanced Raman Scattering Based Immunoassay. Anal Chem 2005, 77, 6147-6154.

(3) Haiss, W.; Thanh, N. T.; Aveyard, J.; Fernig, D. G., Determination of Size and Concentration of Gold Nanoparticles from UV-vis Spectra. Anal Chem 2007, 79, 4215-4221.

(4) Ingamells, C.; Switzer, P., A Proposed Sampling Constant for Use in Geochemical Analysis. Talanta 1973, 20, 547-568. 